\title{
Light elements synthesized in the He-layer and the H-rich envelope of a type II supernova, III -Influence of the nuclear reprocessing after the mixing-
}

\author{
Takashi Yoshida $^{1}$, Hiroyuki Emori ${ }^{2}$, and Kiyoshi Nakazawa ${ }^{2}$ \\ ${ }^{1}$ Department of Physics, Kyushu University, Ropponmatsu, Fukuoka 810-8560, Japan \\ ${ }^{2}$ Department of Earth and Planetary Sciences, Tokyo Institute of Technology, Meguro-ku, Tokyo 152-8551, Japan
}

(Received October 5, 2000; Accepted April 22, 2001)

\begin{abstract}
This is the third paper of a series of our papers, in which we have investigated explosive nucleosynthesis of the $X$-elements ( $\mathrm{Li}, \mathrm{Be}$, and $\mathrm{B})$ and the $\mathrm{CNO}$-elements in supernova explosions. We concentrate here on evaluating the varieties of the isotopic/elemental ratios of the light elements due to the mixing process between the He-layer and the H-rich envelope of a supernova taking account of nuclear reprocessing after the mixing. Almost all of the $X$-elements are influenced strongly by the nuclear reprocessing after the mixing; the $X$-elements produced in the He-layer are decomposed by protons in the H-rich envelope even if the He-component mixes with a bit of the $\mathrm{H}$-component. The $p$-rich isobars of ${ }^{7} \mathrm{Li}$ and ${ }^{11} \mathrm{~B}$, namely ${ }^{7} \mathrm{Be}$ and ${ }^{11} \mathrm{C}$, are not decomposed in the mixture, so that the ${ }^{11} \mathrm{~B} /{ }^{7} \mathrm{Li}$ ratio is determined by the ${ }^{11} \mathrm{C} /{ }^{7} \mathrm{Be}$ ratio after ${ }^{7} \mathrm{Li}$ and ${ }^{11} \mathrm{~B}$ are completely decomposed. On the other hand, the CNO-elements are almost free from the nuclear reprocessing after the mixing. The small ratios of ${ }^{6} \mathrm{Li} /{ }^{7} \mathrm{Li}$ and ${ }^{9} \mathrm{Be} /{ }^{7} \mathrm{Li}$, and the diagram of ${ }^{11} \mathrm{~B} /{ }^{7} \mathrm{Li}-{ }^{12} \mathrm{C} /{ }^{13} \mathrm{C}$ obtained in the previous studies are still valid.
\end{abstract}

\section{Introduction}

Presolar grains have been identified with large isotopic heterogeneities of several orders of magnitude and their large isotopic heterogeneities suggest us that isotopic traces of nucleosynthetic processes remain in these grains (e.g., Zinner, 1998). Until now, some presolar grains have been identified as supernova origin on the basis of the carbon isotopic ratio, the ${ }^{28} \mathrm{Si}$ excess, and the ${ }^{44} \mathrm{Ca}$ excess from the radioactive decay of ${ }^{44} \mathrm{Ti}$ (Amari et al., 1992, 1995; Nittler et al., 1996). However, some of the grains show the other isotopic ratios inconsistent with those predicted from supernova nucleosynthesis theory (Travaglio et al., 1999). Our poor understanding on this problem is due, at least partly, to the lack of theoretical studies of supernova nucleosynthesis which have challenged the comparison between the isotopic ratios of the elements produced in supernovae and those obtained from presolar grains (e.g., Meyer et al., 1995; Travaglio et al., 1999). In most cases, theoretical studies of supernova nucleosynthesis have aimed at the overall element production in supernovae to interpret the solar-system composition (e.g., Woosley et al., 1990; Woosley and Weaver, 1995; Thielemann et al., 1990, 1996).

Our interest is to understand extensively the isotopic/elemental ratios of elements ejected from supernovae and to establish methods for finding presolar grains from a supernova. We have studied the varieties of the isotopic/elemental ratios of the $X$-elements ( $\mathrm{Li}, \mathrm{Be}$, and $\mathrm{B}$ ) and the $\mathrm{CNO}-\mathrm{el}-$ ements synthesized in the He-layer and the H-rich envelope

Copy right (C) The Society of Geomagnetism and Earth, Planetary and Space Sciences (SGEPSS); The Seismological Society of Japan; The Volcanological Society of Japan; The Geodetic Society of Japan; The Japanese Society for Planetary Sciences. of a $16.2 M_{\odot}$ supernova which corresponds to a $20 M_{\odot}$ zeroage main sequence star (Shigeyama and Nomoto, 1990). In Yoshida et al. (2000a) which hereafter is referred to as Paper I, we investigated the influence of the adopted neutrino emission model and presented useful diagrams composed of two kinds of isotopic/elemental ratios. In Yoshida et al. (2000b) which hereafter is referred to as Paper II, we studied the explosive nucleosynthesis using modeled chemical compositions composed of four elements as ${ }^{1} \mathrm{H},{ }^{4} \mathrm{He},{ }^{12} \mathrm{C}$, and ${ }^{16} \mathrm{O}$. Combining probable ranges of the chemical compositions in the presupernova stage, we refined the diagrams. The results of the two papers are summarized as follows:

1) Among the $X$-elements, ${ }^{7} \mathrm{Li}$ and ${ }^{11} \mathrm{~B}$ are synthesized appreciably in the He-layer and the inner H-rich envelope of a supernova and ${ }^{10} \mathrm{~B}$ is also synthesized in the He-layer. The amounts of these elements depend on the adopted neutrino emission model rather than the chemical compositions in the presupernova stage.

2) In the convective He-layer, ${ }^{13} \mathrm{C},{ }^{14} \mathrm{~N},{ }^{15} \mathrm{~N}$, and ${ }^{17} \mathrm{O}$ among the CNO-elements are synthesized during the supernova explosion. The amounts of these elements depend on the neutrino emission model but the varieties are confined within a factor of 10 or so. Furthermore, stable isotopes ${ }^{12} \mathrm{C},{ }^{16} \mathrm{O}$, and ${ }^{18} \mathrm{O}$ keep their amounts in the presupernova stage.

3) In the radiative He-layer and the $\mathrm{H}$-rich envelope where the shock temperature is lower than $1.4 \times 10^{8} \mathrm{~K}$, the $\mathrm{CNO}$-elements are scarcely synthesized during the supernova explosion. 
4) Presolar grains from a supernova are certainly distinguishable by the use of the diagrams of ${ }^{11} \mathrm{~B} /{ }^{7} \mathrm{Li}$ ${ }^{12} \mathrm{C} /{ }^{13} \mathrm{C},{ }^{14} \mathrm{~N} /{ }^{15} \mathrm{~N}-{ }^{12} \mathrm{C} /{ }^{13} \mathrm{C}$, and ${ }^{16} \mathrm{O} /{ }^{17} \mathrm{O}-{ }^{12} \mathrm{C} /{ }^{13} \mathrm{C}$. Additionally, we can say that presolar grains with very small ${ }^{6} \mathrm{Li} /{ }^{7} \mathrm{Li}$ and ${ }^{9} \mathrm{Be} /{ }^{7} \mathrm{Li}$ ratios $\left({ }^{6} \mathrm{Li} /{ }^{7} \mathrm{Li} \lesssim 3 \times 10^{-5}\right.$ and $\left.{ }^{9} \mathrm{Be} /{ }^{7} \mathrm{Li} \lesssim 2 \times 10^{-4}\right)$ are supernova origin.

Other than the neutrino emission and the chemical compositions in the presupernova stage, there is the possibility that the explosive nucleosynthesis is influenced by mixing between layers during the supernova explosion. Some authors pointed out theoretically that the Rayleigh-Taylor instability should take place during supernova explosions (Chevalier, 1976; Ebisuzaki et al., 1989) and, as a result, would lead to large scale mixing in the ejecta (e.g., Hachisu et al., 1990, 1992, 1994; Fryxell et al., 1991; Herant and Woosley, 1994). Further, it is suggested that the convective instability just above a proto-neutron star enables successful explosion of the supernova (Herant et al., 1992; Burrows and Fryxell, 1992; Burrows et al., 1995). Although no silicate grains have been observed in SN 1987A, existence of large scale mixing during the supernova explosion is also supported by observations of light curve, $\gamma$-ray spectra, infrared spectra, and so on, for SN 1987A (e.g., Arnett and Fu, 1989; Fu and Arnett, 1989; Shigeyama and Nomoto, 1990; Wooden, 1997).

Large scale mixing during supernova explosions has influences on our problem in two ways: one is that the mixing brings about a chemical blend between two mixed fluid elements (hereafter such mixing will be called "the mechanical mixing") and the other is that new nucleosynthetic reactions are activated by the mixing and the chemical composition of the mixture varies not only by the mechanical mixing but also by "the nuclear reprocessing after the mixing". In Paper I and Paper II, we have already taken account of the former effect in constructing the diagrams between two isotopic/elemental ratios but not the latter effect. Since the Rayleigh-Taylor instability occurs just after the shock arrival to the $\mathrm{He} / \mathrm{H}$ boundary and develops with the characteristic time of dynamical motion (e.g., Herant and Woosley, 1994), the temperature in the mixture may be high enough to induce new kinds of nucleosynthesis. In this study, we will concentrate ourselves on the study of the nuclear reprocessing after the large-scale mixing between the He-layer and the H-rich envelope.

In Section 2, we describe in short the explosion model of a $16.2 M_{\odot}$ supernova, the nuclear reaction network, and the initial chemical compositions, all of which were constructed in Paper I. Furthermore, we explain how to describe the mixing during the supernova explosion. The mixing is simply modeled by four parameters, i.e., the locations of two fluid elements which will be mixed, the time of mixing, and the mixing ratio between the two fluid elements. In Section 3, we describe in detail, as a typical example, how the abundance of ${ }^{7} \mathrm{Li}$ is influenced by the nuclear reprocessing after the mixing as well as the adopted parameters of the mixing model. In addition, we present briefly influences of the nuclear reprocessing on the abundances of the other light elements comparing with the results in the case of the mechanical mixing. In Section 4, we show the varieties of ${ }^{6} \mathrm{Li} /{ }^{7} \mathrm{Li}$,
${ }^{9} \mathrm{Be} /{ }^{7} \mathrm{Li}$, and ${ }^{11} \mathrm{~B} /{ }^{7} \mathrm{Li}$ ratios which are caused by the nuclear reprocessing after the mixing. We summarize numerical results obtained in this study in Section 5 and construct a diagram of the ratios of ${ }^{11} \mathrm{~B} /{ }^{7} \mathrm{Li}$ and ${ }^{12} \mathrm{C} /{ }^{13} \mathrm{C}$ adding the effect of the nuclear reprocessing after the mixing.

\section{Method of Calculation}

\subsection{Model of supernova explosion}

In this study, we adopt quite the same supernova explosion model as that presented in Paper I. In this model, shock propagation is described on the basis of "the generalized Sedov solution" of a spherically symmetric strong shock wave in the medium with one power law density profile (Sedov, 1959). The power law density profile is fitted to the density structure of 14E1 model written in Shigeyama and Nomoto (1990), i.e., a $16.2 M_{\odot}$ presupernova model which corresponds to a $20 M_{\odot}$ zero-age main sequence star (see figures 2 and 3 in Paper I).

We also make use of our nuclear reaction network, described in Paper I, which consists of 52 species of nuclei from ${ }^{1} \mathrm{H}$ to ${ }^{23} \mathrm{Mg}$. All reactions in this network are compiled in Yoshida (2000). Neutrino-induced reactions (Woosley et al., 1990), so-called the $v$-process, are also included in our reaction network and their reaction rates are adopted from Hoffman and Woosley (1992). For parameters governing the $v$-process, we use the same values as those of Paper I, i.e., temperatures of neutrino flavors are taken to be

$$
\begin{aligned}
& T_{\nu e}=T_{\bar{\nu} e}=4 \mathrm{MeV} / k \text { and } \\
& T_{\nu \mu}=T_{\bar{\nu} \mu}=T_{\nu \tau}=T_{\bar{\nu} \tau}=8 \mathrm{MeV} / k,
\end{aligned}
$$

where $k$ is Boltzmann constant. For the total energy carried by neutrinos, $E_{v}$, and the decay time of neutrino flux, $\tau_{v}$, we put to be the standard values of Paper I, i.e.,

$$
E_{v}=3 \times 10^{53} \mathrm{erg} \text { and } \tau_{v}=3 \mathrm{~s},
$$

in most cases. Only when we construct a diagram between two isotopic ratios in Section 5, we also consider additional four cases (see Paper I):

$$
\begin{aligned}
& \tau_{v}=1 \mathrm{~s} \text { and } 10 \mathrm{~s} \\
& \left(E_{v} \text { is fixed to be } 3 \times 10^{53} \mathrm{erg}\right)
\end{aligned}
$$

and

$$
\begin{aligned}
& E_{\nu}=1 \times 10^{53} \mathrm{erg} \text { and } 6 \times 10^{53} \mathrm{erg} \\
& \left(\tau_{\nu} \text { is fixed to be } 3 \mathrm{~s}\right) .
\end{aligned}
$$

In Paper II, we showed that the amounts of the light elements produced during the supernova explosion actually depend on the initial chemical compositions. However, as long as we choose suitable two isotopic/elemental ratios between them, the ratios are not affected so much by the adopted initial chemical compositions. So, in this study, the initial chemical compositions in the He-layer and the H-rich envelope are taken to be the same as in Paper I (see table 2 and figure 4 in Paper I). 
Table 1. Original locations of the He-component, $M_{m}(\mathrm{He})$, and the H-component, $M_{m}(\mathrm{H})$. These locations are written in terms of the mass coordinate. For the later convenience, we also show the corresponding radial coordinates, $r_{m}(\mathrm{He})$ and $r_{m}(\mathrm{H})$ at the time when the shock front arrives at the He/H boundary. The radial coordinates are written in the unit of $1 \times 10^{9} \mathrm{~cm}$. Note that the He-components at locations 7 and 8 are in the radiative He-layer.

\begin{tabular}{ccccc}
\hline & \multicolumn{2}{c}{ He-component } & \multicolumn{2}{c}{ H-component } \\
\hline & $\begin{array}{c}\text { Location } \\
\left(M_{r} / M_{\odot}\right)\end{array}$ & $\begin{array}{c}\text { Radial coordinate } \\
\left(r_{m}(\mathrm{He}) / 1 \times 10^{9} \mathrm{~cm}\right)\end{array}$ & $\begin{array}{c}\text { Location } \\
\left(M_{r} / M_{\odot}\right)\end{array}$ & $\begin{array}{c}\text { Radial coordinate } \\
\left(r_{m}(\mathrm{He}) / 1 \times 10^{9} \mathrm{~cm}\right)\end{array}$ \\
\hline 1 & 3.80 & 40.0 & 6.50 & 52.7 \\
2 & 4.00 & 41.1 & 6.80 & 62.1 \\
3 & 4.50 & 43.6 & 7.20 & 75.9 \\
4 & 5.00 & 46.0 & 8.00 & 109 \\
5 & 5.50 & 48.4 & 8.80 & 151 \\
6 & 6.00 & 50.57 & & \\
7 & 6.03 & 50.65 & & \\
8 & 6.40 & 52.3 & & \\
\hline
\end{tabular}

\subsection{Model of mixing between the He-layer and the $\mathrm{H}$ - rich envelope}

A progenitor of a type II supernova consists of several layers with different chemical compositions. At each boundary of these layers there is a density gap because of the discontinuity of the mean molecular weight and the continuity of the temperature as well as the pressure at the boundary. The gap at the boundary between the He-layer and the H-rich envelope is expressed in terms of the densities of the top of the He-layer, $\rho_{\mathrm{He}}$, and the bottom of the H-rich envelope, $\rho_{\mathrm{H}}$ as

$$
\frac{\rho_{\mathrm{He}}-\rho_{\mathrm{H}}}{\rho_{\mathrm{He}}+\rho_{\mathrm{H}}}=\frac{\mu_{\mathrm{He}}-\mu_{\mathrm{H}}}{\mu_{\mathrm{He}}+\mu_{\mathrm{H}}}=0.32
$$

where $\mu_{\mathrm{He}}(=1.34)$ and $\mu_{\mathrm{H}}(=0.69)$ are the mean molecular weights in the He-layer and the H-rich envelope, respectively (see section 3.4 in Paper I). Owing to the density gap, these layers become unstable against the RayleighTaylor instability near the boundary after the shock passage (Ebisuzaki et al., 1989). Since fluid elements decelerate behind the shock front, fluid elements of the heavy He-layer feel relatively strong outward inertia force and penetrate into the H-rich envelope. The outward inertia force per unit mass is almost equal to the pressure gradient (Chevalier, 1976).

Recently, some authors have conducted hydrodynamical simulations of the Rayleigh-Taylor instability during supernova explosions (e.g., Hachisu et al., 1990). In their studies, fluid elements in the He-layer penetrate into the H-rich envelope in the form of plumes with mushroom-like structure. However, it is difficult to see detailed microscopic processes of the mixing in the hydrodynamical simulations. So, on the basis of the hydrodynamical simulations of the mixing, we construct the model of the mixing between the Hecomponent and the H-component:

1) Original location of the fluid elements in the He-layer is $M_{m}(\mathrm{He})$, and that in the H-rich envelope is $M_{m}(\mathrm{H})$. Note that the locations are presented in terms of the mass coordinate.

2) The two fluid elements mix each other at $t_{m}$ after the shock front passes the boundary.
3) The mixing ratio between the two fluid components is $x$.

4) The pressure and the temperature of the mixture are determined so as to balance with those of surrounding matter.

For the original locations of the He-component, $M_{m}(\mathrm{He})$, and the H-component, $M_{m}(\mathrm{H})$, we consider eight and five cases, respectively. They are tabulated in Table 1 together with the corresponding radial distances, $r_{m}(\mathrm{He})$ and $r_{m}(\mathrm{H})$, at the time when the shock front arrives at the $\mathrm{He} / \mathrm{H}$ boundary. According to the hydrodynamical simulations, He-components penetrate into the outer region of the H-rich envelope with a mushroom-like feature (e.g., Hachisu et al., 1992; Herant and Woosley, 1994). However, we do not consider such a distant mixing because the nuclear reprocessing after the mixing does not occur practically in such mixing, as will be shown in Section 3 .

The mixing time, $t_{m}$, is chosen as follows:

$$
t_{m}=t_{a}+\tau_{m}
$$

where $t_{a}$ is the time when the shock arrives at the $\mathrm{He} / \mathrm{H}$ boundary and $\tau_{m}$ is the time interval between the shock arrival time and the mixing time. The time interval, $\tau_{m}$, should be of the order of the growth time of the Rayleigh-Taylor instability, $\tau_{\mathrm{RT}}$, given by (e.g., Chandrasekhar, 1981)

$$
\tau_{\mathrm{RT}}=1 / \sqrt{\frac{\rho_{\mathrm{He}}-\rho_{\mathrm{H}}}{\rho_{\mathrm{He}}+\rho_{\mathrm{H}}} \kappa\left|\left(-\frac{1}{\rho}\right) \frac{\partial P}{\partial r}\right|},
$$

where $\kappa$ is the azimuthal wave number of perturbations. In this study we define $\tau_{m}$ as

$$
\tau_{m}=c_{m} \sqrt{\kappa l} \tau_{\mathrm{RT}}
$$

where $c_{m}$ is a numerical parameter. We set $c_{m}$ to be $0.5,1$ (the standard value), 2 , and 5 and set $l$ to be $r_{m}(\mathrm{H})-r_{m}(\mathrm{He})$.

Generally, it is expected that the two components mix with various mixing ratios. So, we choose fifteen different 
mixing ratios, $x$ :

$$
\begin{aligned}
x= & 0,1 \times 10^{-5}, 1 \times 10^{-4}, 1 \times 10^{-3}, 2 \times 10^{-3}, \\
& 5 \times 10^{-3}, 1 \times 10^{-2}, 2 \times 10^{-2}, \\
& 5 \times 10^{-2}, 0.1,0.3,0.5,0.7,0.9,1 .
\end{aligned}
$$

In the above, the case of $x=0$ corresponds to a pure Hecomponent and that of $x=1$ corresponds to a pure $\mathrm{H}$ component.

The pressure of the mixture, $P_{m}$, should become quite the same as that of the surrounding gas of the H-rich envelope. The temperature of the mixture, $T_{m}$, is expected to be nearly equal to that of the surrounding H-rich envelope. So in this study we simply put to be

$$
P_{m}=P_{\mathrm{H}} \quad \text { and } \quad T_{m}=T_{\mathrm{H}} \quad \text { for } \quad t \geq t_{m},
$$

where $P_{\mathrm{H}}$ and $T_{\mathrm{H}}$ are the pressure and the temperature of the surrounding $\mathrm{H}$-rich envelope, respectively. In this case, the density of the mixture is found to be

$$
\rho_{m}=\frac{\mu_{m}}{\mu_{\mathrm{H}}} \rho_{\mathrm{H}} \quad \text { for } \quad t \geq t_{m}
$$

In the above, $\rho_{\mathrm{H}}$ is the density of the surrounding H-rich envelope and the mean molecular weight of the mixture, $\mu_{m}$, is given by

$$
\frac{1}{\mu_{m}}=\frac{1-x}{\mu_{\mathrm{He}}}+\frac{x}{\mu_{\mathrm{H}}},
$$

where $x$ is the mixing ratio mentioned above.

\section{Nuclear Reprocessing Due to the Mixing \\ 3.1 Typical example of the nuclear reprocessing-Case of ${ }^{7} \mathbf{L i}$}

In Fig. 1, time variation of the abundances of ${ }^{7} \mathrm{Li}$ and its unstable isobar, ${ }^{7} \mathrm{Be}$, is shown in the case of $x=0.1$, $c_{m}=1, M_{m}(\mathrm{He})=5.5 M_{\odot}$, and $M_{m}(\mathrm{H})=6.5 M_{\odot}($ which corresponds to the bottom of the H-rich envelope). Furthermore, $t_{a}$ and $t_{m}$ are the time of the shock arrival and the time of the mixing, respectively. Before the shock arrival, both ${ }^{7} \mathrm{Li}$ and ${ }^{7} \mathrm{Be}$ are produced through the $\nu$-process in the Hecomponent; the main production paths of ${ }^{7} \mathrm{Li}$ and ${ }^{7} \mathrm{Be}$ are ${ }^{4} \mathrm{He}\left(v, v^{\prime} p\right){ }^{3} \mathrm{H}(\alpha, \gamma){ }^{7} \mathrm{Li}$ and ${ }^{4} \mathrm{He}\left(v, v^{\prime} n\right){ }^{3} \mathrm{He}(\alpha, \gamma){ }^{7} \mathrm{Be}$, respectively. At the same time, ${ }^{7} \mathrm{Be}$ is decomposed through ${ }^{7} \mathrm{Be}(n, p){ }^{7} \mathrm{Li}$. In the $\mathrm{H}$-component, ${ }^{7} \mathrm{Be}$ is formed through the same production process as in the He-component but ${ }^{7} \mathrm{Li}$ keeps its initial abundance. After the shock arrival to the Hecomponent, ${ }^{7} \mathrm{Li}$ and ${ }^{7} \mathrm{Be}$ are produced explosively through ${ }^{3} \mathrm{H}(\alpha, \gamma){ }^{7} \mathrm{Li}$ and ${ }^{3} \mathrm{He}(\alpha, \gamma){ }^{7} \mathrm{Be}$. The abundance of ${ }^{7} \mathrm{Li}$ in the He-component attains to $4 \times 10^{-7}$. In the H-component, ${ }^{7} \mathrm{Be}$ is produced explosively after the shock arrival but the abundance of ${ }^{7} \mathrm{Li}$ rapidly decreases owing to decomposition by protons.

As soon as the He-component and the H-component mix each other at the time, $t_{m}$, the abundance of ${ }^{7} \mathrm{Li}$ in the mixture decreases rapidly; ${ }^{7} \mathrm{Li}$ which is abundant in the Hecomponent is decomposed by protons which are abundant in the H-component through ${ }^{7} \mathrm{Li}(p, \alpha \gamma){ }^{4} \mathrm{He}$. However, $p$-rich isobar, ${ }^{7} \mathrm{Be}$, is not decomposed even after the mixing. Thus,

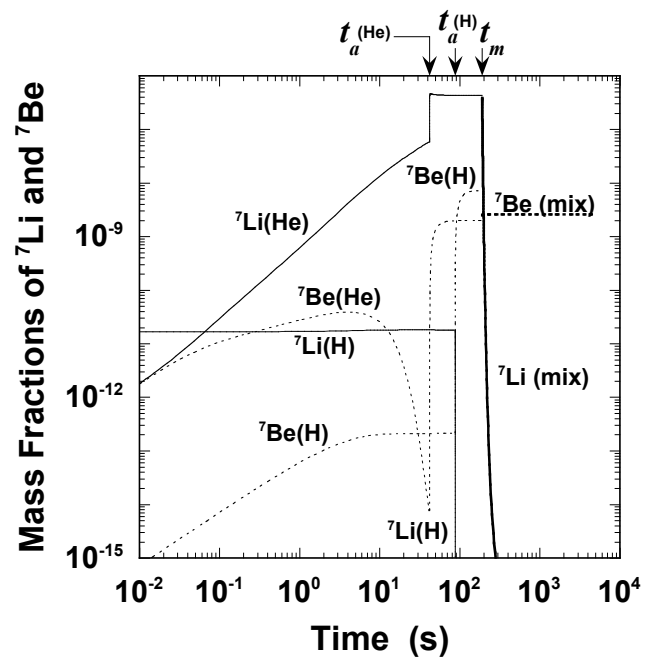

Fig. 1. Time variation of the abundances of ${ }^{7} \mathrm{Li}$ (solid lines) and ${ }^{7} \mathrm{Be}$ (dotted lines). Symbols $\mathrm{He}$ and $\mathrm{H}$ in parentheses denote of the He-component at $M_{m}(\mathrm{He})=5.5 M_{\odot}$ and of the H-component at $M_{m}(\mathrm{H})=6.5 M_{\odot}$, respectively. Bold lines are of the mixture with the mixing ratio, $x$, equal to 0.1 .

the final abundance of ${ }^{7} \mathrm{Li}$ is determined by the abundance of ${ }^{7} \mathrm{Be}$ remained in the mixture.

Next, the final abundances of ${ }^{7} \mathrm{Li}$ with different locations of the He-component, $M_{m}(\mathrm{He})$, and the mixing ratio, $x$, are shown in Fig. 2. Figures 2(a) and (b) are in the cases of $M_{m}(\mathrm{He})=3.8 M_{\odot}$ (the innermost region of the He-layer $)$ and $6.0 M_{\odot}$ (the outermost region), respectively. The original location of the H-component, $M_{m}(\mathrm{H})$, is fixed to be 6.5 $M_{\odot}$ in both figures. Solid line and dotted line denote the final abundances of ${ }^{7} \mathrm{Li}$ and ${ }^{7} \mathrm{Be}$, respectively, in the case of the mechanical mixing (i.e., the case that the nuclear reprocessing is not considered). In Fig. 2(a), the final abundance of ${ }^{7} \mathrm{Li}$ in the mixture agrees with the solid line, i.e., the nuclear reprocessing after the mixing does not occur practically. This is due to the fact that, as shown in Paper I, ${ }^{7} \mathrm{Li}$ is originally produced as ${ }^{7} \mathrm{Be}$ in the inner region of the Helayer and that ${ }^{7} \mathrm{Be}$ is not decomposed by protons even after the mixing in this case (see Fig. 1).

As seen from Fig. 2(b), on the other hand, the mixture with the He-component from the outer region, namely $M_{m}(\mathrm{He}) \gtrsim 5.0 M_{\odot}$, the final abundance of ${ }^{7} \mathrm{Li}$ behaves differently from the previous case. When the mixing ratio, $x$, is smaller than $1 \times 10^{-3},{ }^{7} \mathrm{Li}$ is not decomposed because of a shortage of proton abundance. When $2 \times 10^{-3} \lesssim x \lesssim$ $2 \times 10^{-2}$, the abundance of ${ }^{7} \mathrm{Li}$ decreases with an increase in the mixing ratio; this is brought about by the decomposition of ${ }^{7} \mathrm{Li}$ through ${ }^{7} \mathrm{Li}(p, \alpha \gamma)^{4} \mathrm{He}$ in the mixture. In the case of $x \gtrsim 2 \times 10^{-2},{ }^{7} \mathrm{Li}$ is completely decomposed and the final abundance of ${ }^{7} \mathrm{Li}$ is determined by that of ${ }^{7} \mathrm{Be}$ unprocessed by the mixing. In the case of the mixing with the He-component from the radiative He-layer, the behavior of the abundance of ${ }^{7} \mathrm{Li}$ in the mixture is similar to this.

Figure 2(b) also shows the influence of the mixing time, $c_{m}$ on the final abundance of ${ }^{7} \mathrm{Li}$. With an increase in $c_{m}$, the nuclear reprocessing after the mixing becomes less effective because the temperature becomes lower at the moment of 

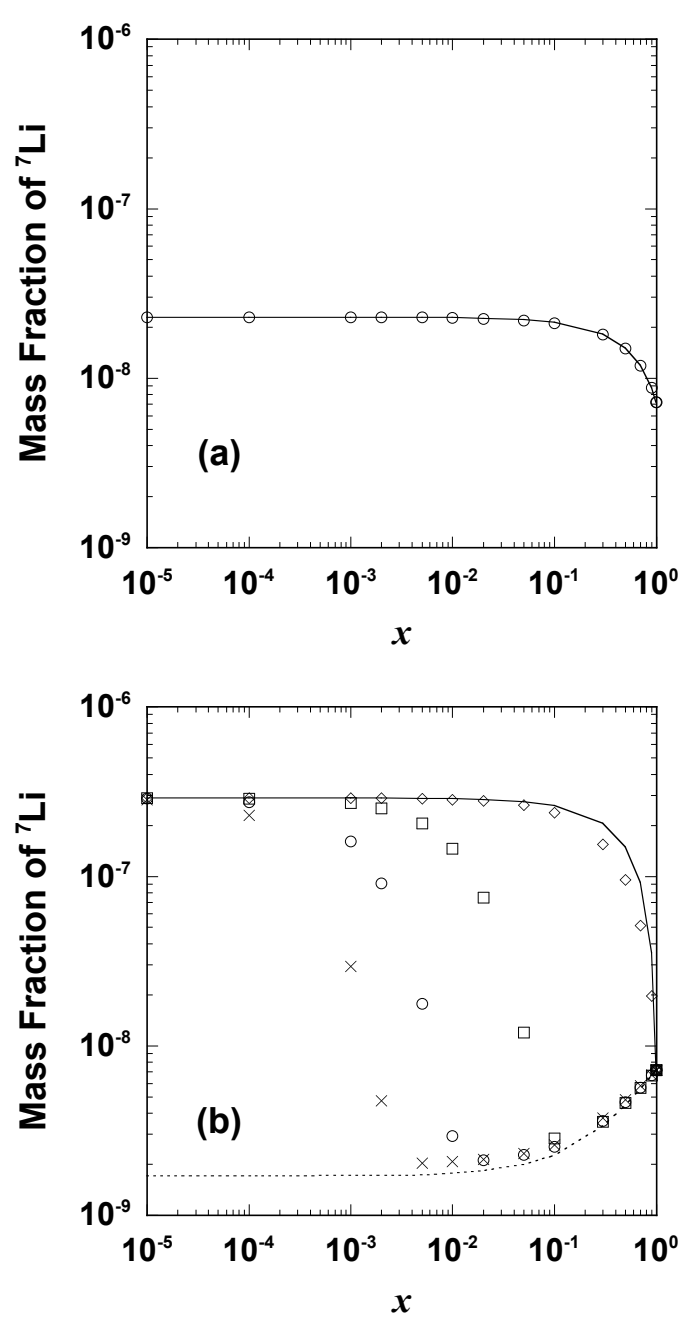

Fig. 2. The final abundances of ${ }^{7} \mathrm{Li}$ in the mixture in the cases of $M_{m}(\mathrm{He})=3.8 M_{\odot}\left(\right.$ panel (a)) and $6.0 M_{\odot}($ panel (b)). The location of the H-component is fixed to be $M_{m}(\mathrm{H})=6.5 M_{\odot}$. The horizontal axis denotes the mixing ratio, $x$. Open circles are the abundances of ${ }^{7} \mathrm{Li}$ in the mixtures in the case of $c_{m}=1$ (see Subsection 2.2). In panel (b), crosses, squares, and diamonds are the abundances in the cases of $c_{m}=0.5,2$, and 5 , respectively. Solid line and dotted line show the final abundance of ${ }^{7} \mathrm{Li}$ and the abundance of ${ }^{7} \mathrm{Be}$ produced during the explosion, respectively, in the case of the mechanical mixing.

the mixing as $c_{m}$ becomes larger, namely the mixing time $\tau_{m}$ does, too. In the case of $c_{m}=5$, the abundance of ${ }^{7} \mathrm{Li}$ almost agrees with the solid line, i.e., ${ }^{7} \mathrm{Li}$ is scarcely decomposed by protons in this case.

In Fig. 3, we show the abundances of ${ }^{7} \mathrm{Li}$ in the mixtures between the H-component with various original locations of $M_{m}(\mathrm{H})$ and the He-component with $M_{m}(\mathrm{He})=6.0 M_{\odot}$. For large $M_{m}(\mathrm{H}),{ }^{7} \mathrm{Li}$ suffers the nuclear reprocessing in the mixture at large mixing ratio, i.e., the nuclear reprocessing becomes less effective when $M_{m}(\mathrm{H})$ is large. In the case of $M_{m}(\mathrm{H})=6.8 M_{\odot},{ }^{7} \mathrm{Li}$ is completely decomposed and only ${ }^{7} \mathrm{Be}$ remains when $x \gtrsim 0.3$. On the other hand, in the cases of $M_{m}(\mathrm{H})=8.0 M_{\odot}$ and $8.8 M_{\odot}$, the abundance of ${ }^{7} \mathrm{Li}$ is almost equal to the corresponding abundance in the case of the mechanical mixing. The abundances of the other light elements behave similarly in such mixing between the fluid elements which are at a long distance at the pre-mixing

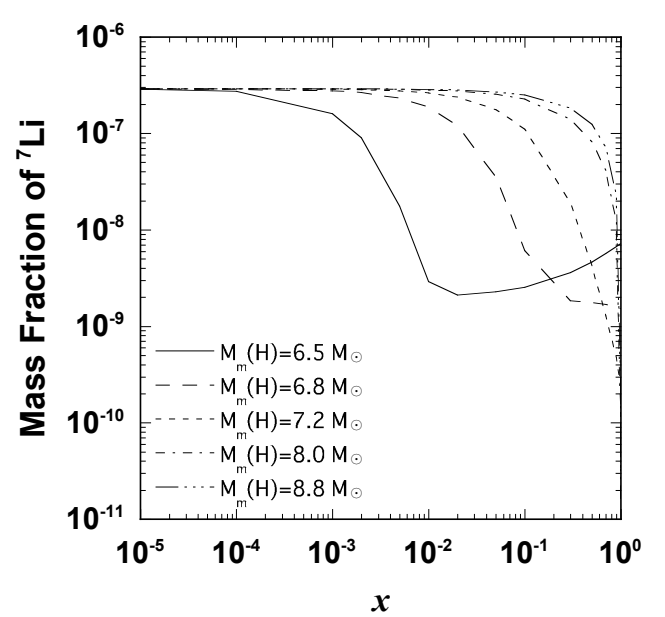

Fig. 3. The same as Fig. 2 but in the cases with various $M_{m}(\mathrm{H})$. The location of the He-component is $M_{m}(\mathrm{He})=6.0 M_{\odot}$ and $c_{m}$ is fixed to be 1 .

stage. Thus, we do not need to consider such mixing with $M_{m}(\mathrm{H}) \gtrsim 8.8 M_{\odot}$, which hereafter we call the distant mixing.

\subsection{Reprocessing on the other $X$-elements}

3.2.1 ${ }^{11} \mathbf{B}$ We will consider the nuclear reprocessing on ${ }^{11} \mathrm{~B}$ after the mixing. The abundances of ${ }^{11} \mathrm{~B}$ in the mixture in the cases of $M_{m}(\mathrm{He})=3.8 M_{\odot}$ and $6.0 M_{\odot}$ are presented in Figs. 4(a) and (b), respectively $\left(M_{m}(\mathrm{H})\right.$ is fixed to be $\left.6.5 M_{\odot}\right)$. In these figures, the abundance of ${ }^{11} \mathrm{~B}$ decreases with an increase in the mixing ratio, $x$, and converges to the abundance of ${ }^{11} \mathrm{C}$ in the mixture at the shock arrival to the $\mathrm{He} / \mathrm{H}$ boundary shown by the dotted line, as same as the case of ${ }^{7} \mathrm{Li}$. As seen in Paper I, ${ }^{11} \mathrm{~B}$ is also formed as the decay product of ${ }^{11} \mathrm{C}$ as in the case of ${ }^{7} \mathrm{Li}$ which is also produced from ${ }^{7} \mathrm{Be}$. The production reactions are ${ }^{7} \mathrm{Li}(\alpha, \gamma){ }^{11} \mathrm{~B}$ and ${ }^{12} \mathrm{C}\left(\nu, v^{\prime} p\right){ }^{11} \mathrm{~B}$ for ${ }^{11} \mathrm{~B}$ and ${ }^{7} \mathrm{Be}(\alpha, \gamma){ }^{11} \mathrm{C}$ and ${ }^{12} \mathrm{C}\left(\nu, v^{\prime} n\right){ }^{11} \mathrm{C}$ for ${ }^{11} \mathrm{C}$. Then, although ${ }^{11} \mathrm{~B}$ is decomposed by protons in the mixture, its $p$-rich isobar, ${ }^{11} \mathrm{C}$, remains even after the mixing and decays into ${ }^{11} \mathrm{~B}$. In the case of $M_{m}(\mathrm{He})=6.0 M_{\odot},{ }^{11} \mathrm{~B}$ is completely decomposed when $x$ is larger than $2 \times 10^{-2}$. On the other hand, the mixing ratio more than 0.3 is needed to decompose ${ }^{11} \mathrm{~B}$ completely in the case of $M_{m}(\mathrm{He})=3.8$ $M_{\odot}$. This is because it takes longer time to mix and the maximum temperature in the mixture is lower in the latter case than in the former.

3.2.2 ${ }^{10} \mathrm{~B} \quad$ Figure 5 shows the abundance of ${ }^{10} \mathrm{~B}$ in the mixture between the He-component $\left(M_{m}(\mathrm{He})=6.0 M_{\odot}\right)$ and the H-component $\left(M_{m}(\mathrm{H})=6.5 M_{\odot}\right)$. Note that there is no ${ }^{10} \mathrm{~B}$ in the $\mathrm{H}$-component as presented in Paper I. When $x \lesssim 1 \times 10^{-3}$, the final abundance of ${ }^{10} \mathrm{~B}$ is almost equal to that of the mechanical mixing (which is shown by the solid line in Fig. 5). On the other hand, when $x \gtrsim 1 \times 10^{-3}$, the abundance of ${ }^{10} \mathrm{~B}$ becomes small compared with that of the mechanical mixing. This is apparently because the nuclear reprocessing on ${ }^{10} \mathrm{~B}$ occurs, i.e., ${ }^{10} \mathrm{~B}$ is decomposed through ${ }^{10} \mathrm{~B}(p, \alpha){ }^{7} \mathrm{Be}$ in the mixture. Differently from the cases of ${ }^{7} \mathrm{Li}$ and ${ }^{11} \mathrm{~B},{ }^{10} \mathrm{~B}$ has no $p$-rich isobars which are not decomposed by protons. 

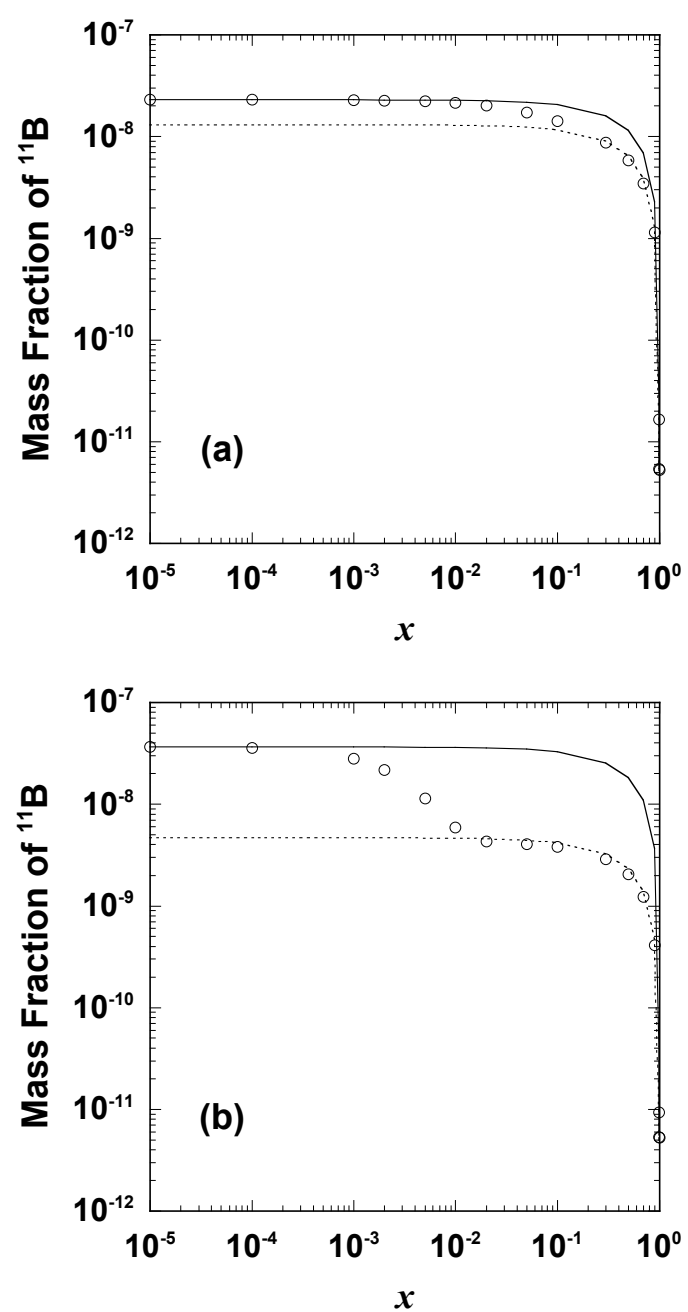

Fig. 4. The same as Fig. 2, but for the final abundances of ${ }^{11} \mathrm{~B}$ in the cases of $M_{m}(\mathrm{He})=3.8 M_{\odot}\left(\right.$ panel (a)) and $6.0 M_{\odot}($ panel (b)). The location of the H-component is fixed to be $M_{m}(\mathrm{H})=6.5 M_{\odot}$ and $c_{m}$ is fixed to be 1. Solid line and dotted line show the final abundances of ${ }^{11} \mathrm{~B}$ and ${ }^{11} \mathrm{C}$, respectively, in the case of the mechanical mixing at the shock arrival to the $\mathrm{He} / \mathrm{H}$ boundary.

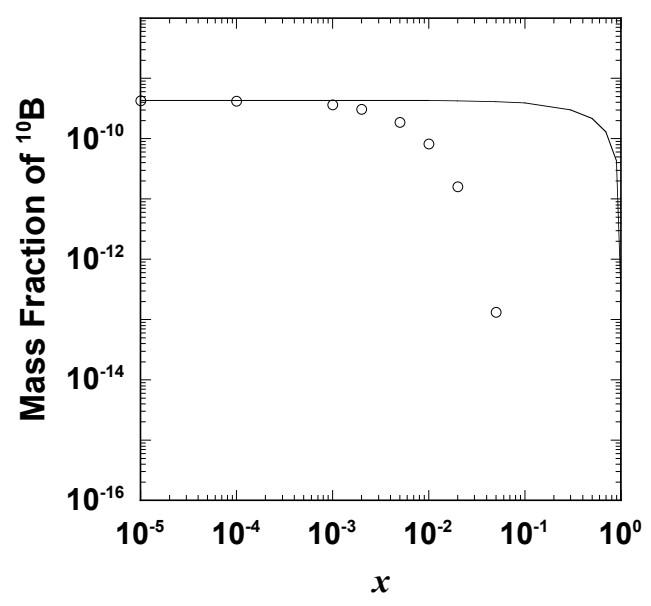

Fig. 5. The final abundances of ${ }^{10} \mathrm{~B}$ (open circles) in the mixture between the He-component at $M_{m}(\mathrm{He})=6.0 M_{\odot}$ and the H-component at $M_{m}(\mathrm{H})=6.5 M_{\odot}\left(c_{m}=1\right)$. Solid line shows the abundance in the case of the mechanical mixing.
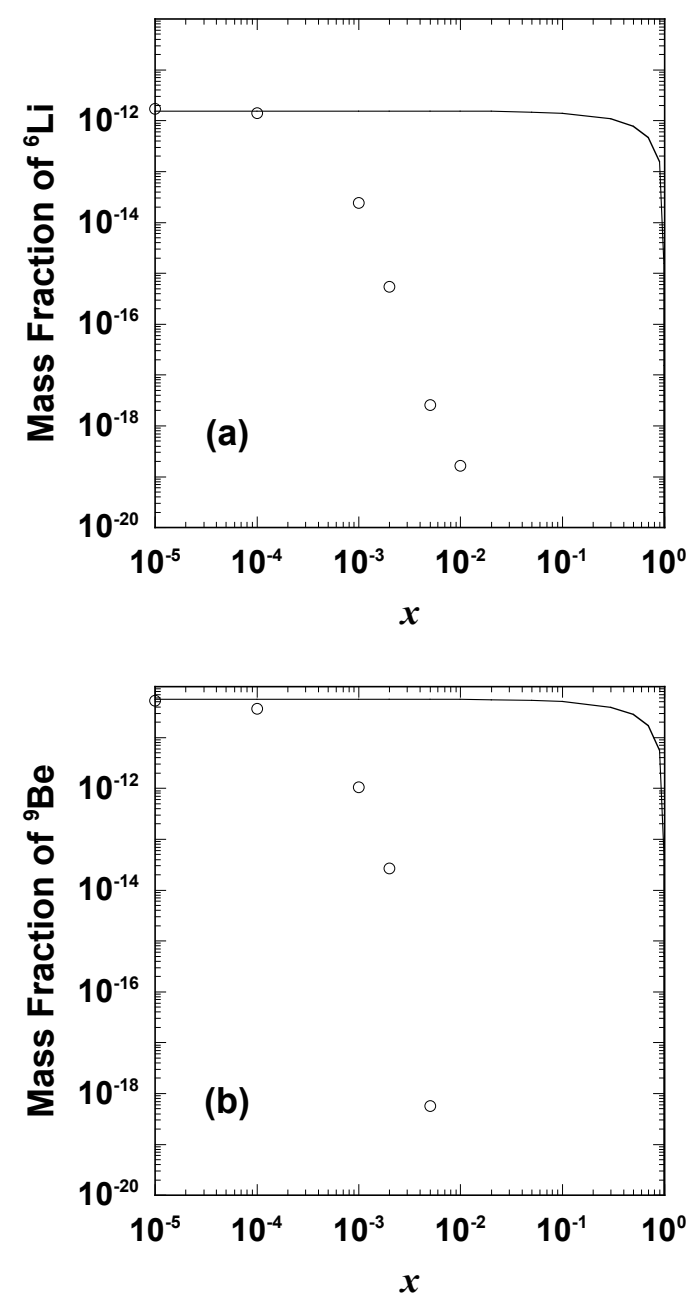

Fig. 6. The same as Fig. 5, but for the abundances of ${ }^{6} \mathrm{Li}$ (panel (a)) and ${ }^{9} \mathrm{Be}($ panel (b)).

3.2.3 ${ }^{6} \mathbf{L i}$ and ${ }^{9} \mathbf{B e} \quad$ In Figs. 6(a) and (b) the abundances of ${ }^{6} \mathrm{Li}$ and ${ }^{9} \mathrm{Be}$ are shown, respectively, in the mixture between the He-component at $M_{m}(\mathrm{He})=6.0 M_{\odot}$ and the H-component at $M_{m}(\mathrm{H})=6.5 M_{\odot}$. As seen from these figures, both ${ }^{6} \mathrm{Li}$ and ${ }^{9} \mathrm{Be}$ are influenced strongly by the nuclear reprocessing even if the mixing ratio is as small as $x \simeq 1 \times 10^{-3}$. In this case ${ }^{6} \mathrm{Li}$ is decomposed through ${ }^{6} \mathrm{Li}(p, \alpha){ }^{3} \mathrm{He}$ and ${ }^{9} \mathrm{Be}$ is decomposed through ${ }^{9} \mathrm{Be}(p, \alpha){ }^{6} \mathrm{Li}$ and ${ }^{9} \operatorname{Be}(p, d \alpha)^{4} \mathrm{He}$.

We showed in Paper I and Paper II that both ${ }^{6} \mathrm{Li}$ and ${ }^{9} \mathrm{Be}$ are not produced in the inner region of the He-layer $\left(M_{r} \lesssim 5.2 M_{\odot}\right)$. In this study, we confirm again that ${ }^{6} \mathrm{Li}$ and ${ }^{9} \mathrm{Be}$ are not produced even in the mixture between the $\mathrm{He}$-component and the $\mathrm{H}$-component. Thus, the abundances of ${ }^{6} \mathrm{Li}$ and ${ }^{9} \mathrm{Be}$ are small except outer region of the He-layer and become extremely small in the mixture contaminated by only small fraction of the H-component.

\subsection{Reprocessing on the CNO-elements}

The abundances of ${ }^{13} \mathrm{C}$ are illustrated in Fig. 7 in the cases where the He-components at $M_{m}(\mathrm{He})=3.8,5.0,6.0$, and $6.4 M_{\odot}$ mix with the H-component at $M_{m}(\mathrm{H})=6.5$ $M_{\odot}$. The parameter $c_{m}$ is taken to be 1 . Four lines denote the corresponding abundances obtained by the mechanical mixing. From this figure we readily see that ${ }^{13} \mathrm{C}$ is al- 


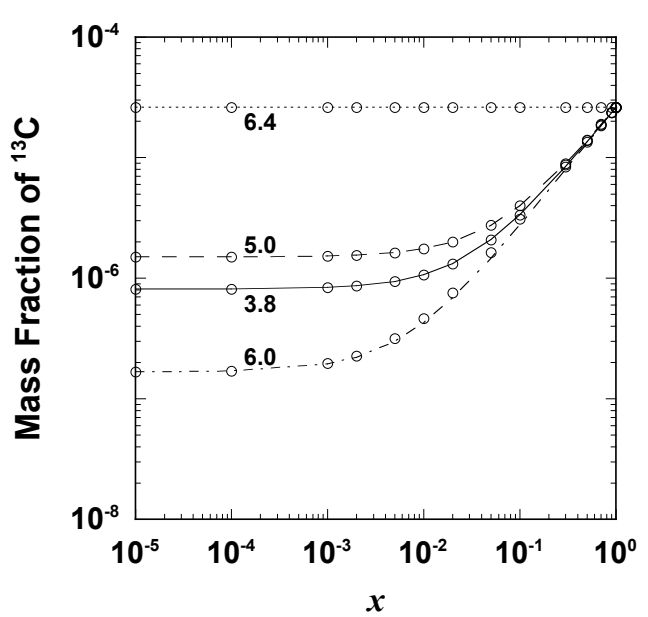

Fig. 7. The final abundances of ${ }^{13} \mathrm{C}$ (open circles) in the mixture in the cases of $M_{m}(\mathrm{He})=3.8 M_{\odot}, 5.0 M_{\odot}, 6.0 M_{\odot}$, and $6.4 M_{\odot}\left(M_{m}(\mathrm{H})\right.$ is fixed to be $6.5 M_{\odot}$ ). Lines show the corresponding abundances of the mechanical mixing and the number attached to each line denotes the location of the He-component.

most perfectly free from the nuclear reprocessing after the mixing, i.e., the ${ }^{13} \mathrm{C}$ abundance is determined by the mechanical mixing. Speaking in detail, only in the case of $M_{m}(\mathrm{He})=6.0 M_{\odot}$ and $c_{m}$ equal to 0.5 , the temperature of the mixture is relatively high and a bit of increment of ${ }^{13} \mathrm{C}$ (through $\left.{ }^{12} \mathrm{C}(p, \gamma){ }^{13} \mathrm{~N}\left(\mathrm{e}^{+} v_{\mathrm{e}}\right)^{13} \mathrm{C}\right)$ is observed after the mixing. However, in all cases where $c_{m} \geq 1$ we see no apparent changes of the ${ }^{13} \mathrm{C}$ abundance. Furthermore, the other choice of $M_{m}(\mathrm{H})$ does not change the above result on ${ }^{13} \mathrm{C}$ since such a choice gives the temperature of the mixture lower than that in the case of Fig. 7.

We observe no nuclear reprocessing after the mixing for the other CNO-elements, including ${ }^{15} \mathrm{~N}$ which seems to be fragile to protons (oppositely, ${ }^{15} \mathrm{~N}$ is slightly produced in the case of $c_{m}=0.5$ ). Thus, we can say that the abundances of the CNO-elements are determined by the $v$-process and explosive nucleosynthesis and are free from the nuclear reprocessing after the mixing, except the mechanical mixing, even if there occurs large scale mixing during the supernova explosion.

\section{Effects of Mixing on the Isotopic Ratios of the $X$-Elements}

\subsection{The ${ }^{6} \mathrm{Li} /{ }^{7} \mathrm{Li}$ and ${ }^{9} \mathrm{Be} /{ }^{7} \mathrm{Li}$ ratios}

The diagram of ${ }^{6} \mathrm{Li} /{ }^{16} \mathrm{O}-{ }^{7} \mathrm{Li} /{ }^{16} \mathrm{O}$ ratios is presented in Fig. 8(a) which is the same form as that in figure 8(a) in Paper I. Hereafter, we present the number ratio of two species as an abundance ratio. Markers linked by the dashed line are the ratios in the mixtures of which He-components come from the same location in the He-layer. The ${ }^{6} \mathrm{Li} /{ }^{16} \mathrm{O}$ ratio is decreased more drastically than the ${ }^{7} \mathrm{Li} /{ }^{16} \mathrm{O}$ ratio by the mixing irrelevant to the mixing ratio and to the location of the He-component. This is due, of course, to the fact that ${ }^{6} \mathrm{Li}$ is more fragile than ${ }^{7} \mathrm{Li}$ to the irradiation of protons followed by the mixing. We see that all markers linked with dashed lines are below the bold line, i.e., the ${ }^{6} \mathrm{Li} /{ }^{7} \mathrm{Li}$ ratio is always smaller than $3 \times 10^{-5}$ which is the maximum value found in Paper I and Paper II. The diagram of ${ }^{9} \mathrm{Be} /{ }^{16} \mathrm{O}$ -
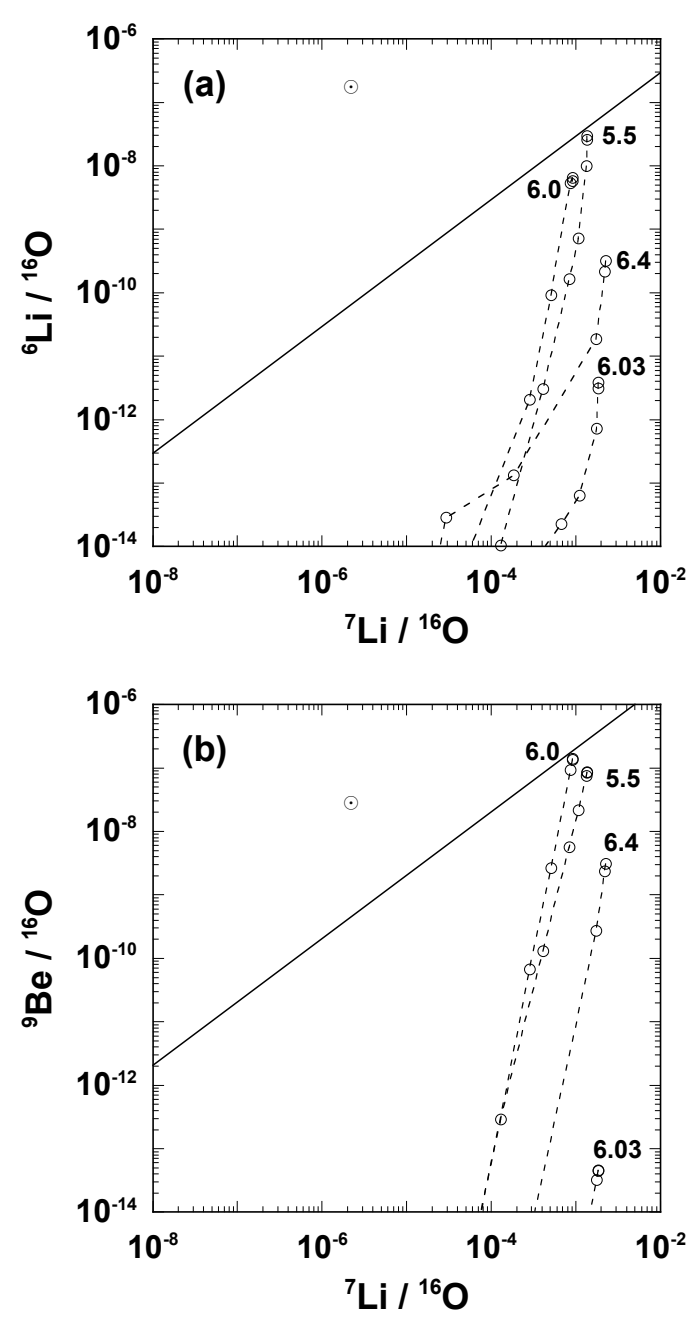

Fig. 8. Diagrams between ${ }^{6} \mathrm{Li} /{ }^{16} \mathrm{O}$ and ${ }^{7} \mathrm{Li} /{ }^{16} \mathrm{O}$ number ratios (panel (a)) and between ${ }^{9} \mathrm{Be} /{ }^{16} \mathrm{O}$ and ${ }^{7} \mathrm{Li} /{ }^{16} \mathrm{O}$ number ratios (panel (b)). Markers connected by the dashed line show the ratios in the mixture with various mixing ratios, $x$. Original locations of the He-components, $M_{m}(\mathrm{He})$, are labeled by attached annotation. Solid lines indicate the ${ }^{6} \mathrm{Li} /{ }^{7} \mathrm{Li}$ ratio equal to $3 \times 10^{-5}$ (panel (a)) and the ${ }^{9} \mathrm{Be} / 7 \mathrm{Li}$ ratio equal to $2 \times 10^{-4}$ (panel (b)). These are the maximum values found in Paper I and Paper II. For comparison we show the ratios of the solar-system composition by $\odot$.

${ }^{7} \mathrm{Li} /{ }^{16} \mathrm{O}$ ratios is presented in Fig. 8(b). Similarly to the case of ${ }^{6} \mathrm{Li} /{ }^{16} \mathrm{O}$, the ${ }^{9} \mathrm{Be} /{ }^{16} \mathrm{O}$ ratio is decreased more steeply than the ${ }^{7} \mathrm{Li} /{ }^{16} \mathrm{O}$ ratio by the mixing and is always smaller than $2 \times 10^{-4}$ which is the maximum value evaluated in Paper I and Paper II. Consequently, the main conclusion obtained in Paper I and Paper II that presolar grains with ${ }^{6} \mathrm{Li} /{ }^{7} \mathrm{Li}$ ratio less than $3 \times 10^{-5}$ and with ${ }^{9} \mathrm{Be} /{ }^{7} \mathrm{Li}$ ratio less than $2 \times 10^{-4}$ should be supernova origin is not altered even if the nuclear reprocessing after the mixing is considered.

\subsection{The ${ }^{11} \mathrm{~B} /{ }^{7} \mathrm{Li}$ ratio}

The final ratio of ${ }^{11} \mathrm{~B} /{ }^{7} \mathrm{Li}$ is influenced by the mixing in a complicated manner in spite of the similar behavior between ${ }^{7} \mathrm{Li}$ and ${ }^{11} \mathrm{~B}$ (see the previous section). The reason is that ${ }^{7} \mathrm{Li}$ and ${ }^{11} \mathrm{~B}$ are easily decomposed by protons but their $p$-rich isobars, ${ }^{7} \mathrm{Be}$ and ${ }^{11} \mathrm{C}$, which decay respectively to ${ }^{7} \mathrm{Li}$ and ${ }^{11} \mathrm{~B}$, are not.

In Fig. 9, the ${ }^{11} \mathrm{~B} /{ }^{7} \mathrm{Li}$ ratio is shown as a function of the mixing ratio, $x$; panels (a), (b), and (c) are of $M_{m}(\mathrm{He})=3.8$ 

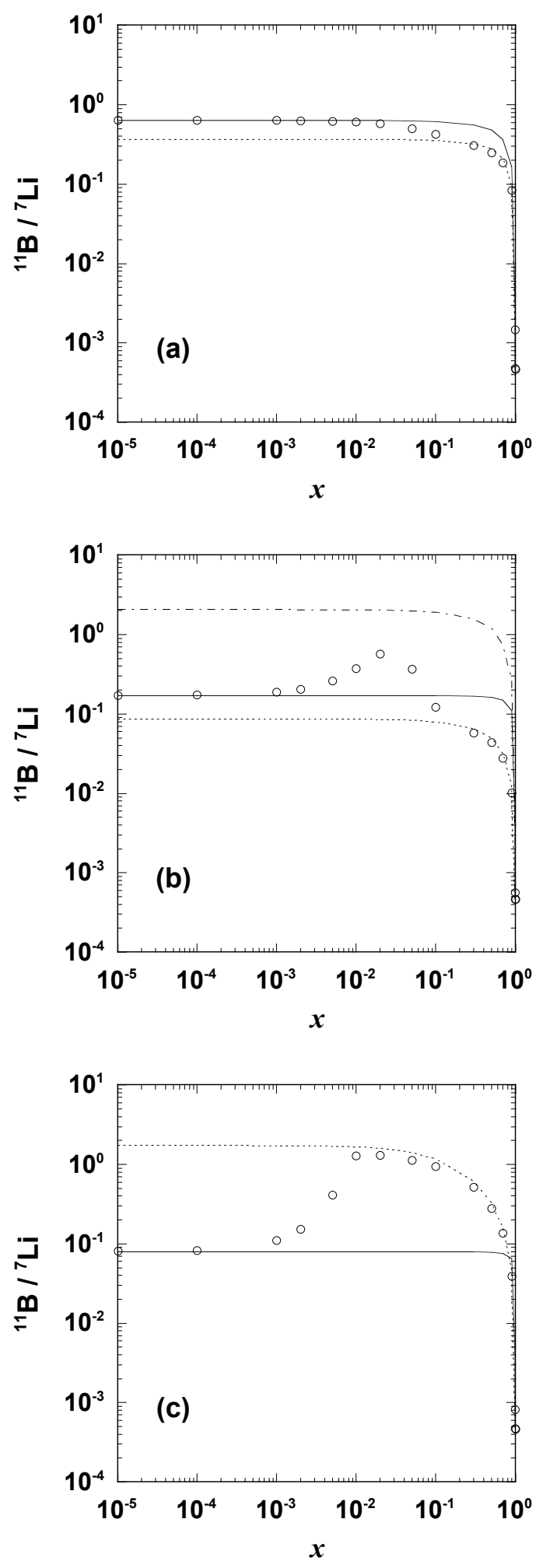

Fig. 9. The final ${ }^{11} \mathrm{~B} /{ }^{7} \mathrm{Li}$ ratios (open circles) in the mixture in the cases of $M_{m}(\mathrm{He})=3.8 M_{\odot}\left(\right.$ panel (a)), 5.0 $M_{\odot}$ (panel (b)), and $6.0 M_{\odot}$ (panel (c)) $\left(M_{m}(\mathrm{H})=6.5 M_{\odot}\right)$. Solid line and dotted line show the mixing lines of ${ }^{11} \mathrm{~B} /{ }^{7} \mathrm{Li}$ and ${ }^{11} \mathrm{C} /{ }^{7} \mathrm{Be}$ at the shock arrival to the $\mathrm{He} / \mathrm{H}$ boundary, respectively. Dash-dotted line in panel (b) shows the ${ }^{11} \mathrm{~B} /{ }^{7} \mathrm{Be}$ ratio of the mechanical mixing.
$M_{\odot}, 5.0 M_{\odot}$, and $6.0 M_{\odot}$, respectively. The location of the H-component, $M_{m}(\mathrm{H})$, is fixed to be $6.5 M_{\odot}$. In the case of $M_{m}(\mathrm{He})=3.8 M_{\odot}($ see Fig. $9(\mathrm{a}))$, the ${ }^{11} \mathrm{~B} /{ }^{7} \mathrm{Li}$ ratio coincides with the solid line when $x \lesssim 2 \times 10^{-2}$ and with the dotted line when $x \gtrsim 0.3$; the former is the mixing line of ${ }^{11} \mathrm{~B} /{ }^{7} \mathrm{Li}$ and the latter is that of ${ }^{11} \mathrm{C} /{ }^{7} \mathrm{Be}$. In the case of $x \lesssim 2 \times 10^{-2}$, neither ${ }^{7} \mathrm{Li}$ nor ${ }^{11} \mathrm{~B}$ is decomposed in the mixture. On the other hand, in the case of $x \gtrsim 2 \times 10^{-2}$ only ${ }^{7} \mathrm{Be}$ synthesized before the mixing remains in the mixture (see Fig. 2(a)), but both ${ }^{7} \mathrm{Li}$ and ${ }^{11} \mathrm{~B}$ are decomposed. As mentioned before, ${ }^{7} \mathrm{Be}$ outnumbers ${ }^{7} \mathrm{Li}$ before the mixing. So, the ${ }^{11} \mathrm{~B} /{ }^{7} \mathrm{Li}$ ratio runs along the mixing line of the ${ }^{11} \mathrm{~B} /{ }^{7} \mathrm{Be}$ ratio when $x \lesssim 2 \times 10^{-2}$ and of the ${ }^{11} \mathrm{C} /{ }^{7} \mathrm{Be}$ ratio when $x \gtrsim 0.3$.

In the case of $M_{m}(\mathrm{He})=5.0 M_{\odot}$, the ${ }^{11} \mathrm{~B} /{ }^{7} \mathrm{Li}$ ratio becomes larger than not only that of the mechanical mixing but also the corresponding ${ }^{11} \mathrm{C} /{ }^{7} \mathrm{Be}$ ratio presented by the dash-dotted line when $1 \times 10^{-3} \lesssim x \lesssim 0.1$ (see Fig. 9(b)). Note here that, although both ${ }^{7} \mathrm{Li}$ and ${ }^{11} \mathrm{~B}$ are decomposed by protons, ${ }^{7} \mathrm{Li}$ is more fragile to the irradiation of protons compared with ${ }^{11} \mathrm{~B}$. When $x \lesssim 3 \times 10^{-2},{ }^{7} \mathrm{Li}$ is decomposed (but ${ }^{11} \mathrm{~B}$ is not) and the ${ }^{11} \mathrm{~B} /{ }^{7} \mathrm{Li}$ ratio becomes larger than the ${ }^{11} \mathrm{~B} /{ }^{7} \mathrm{Li}$ ratio of the mechanical mixing. With a further increase in the mixing ratio, ${ }^{11} \mathrm{~B}$ starts to be decomposed, so that the ${ }^{11} \mathrm{~B} /{ }^{7} \mathrm{Li}$ ratio becomes small. For $x \gtrsim 0.1$, the ${ }^{11} \mathrm{~B} /{ }^{7} \mathrm{Li}$ ratio reduces to the ${ }^{11} \mathrm{C} /{ }^{7} \mathrm{Be}$ ratio of the mechanical mixing because ${ }^{7} \mathrm{Li}$ and ${ }^{11} \mathrm{~B}$ are decomposed almost completely. The ratio of ${ }^{11} \mathrm{~B} /{ }^{7} \mathrm{Li}$ is always smaller than the ${ }^{11} \mathrm{~B} /{ }^{7} \mathrm{Be}$ ratio of the mechanical mixing.

In the case of $M_{m}(\mathrm{He})=6.0 M_{\odot}$, we have a behavior of the ${ }^{11} \mathrm{~B} /{ }^{7} \mathrm{Li}$ ratio different from the previous two cases, as shown in Fig. 9(c), because the ${ }^{11} \mathrm{C} /{ }^{7} \mathrm{Be}$ ratio of the $\mathrm{He}$ component (about 2) is greater than the ${ }^{11} \mathrm{~B} /{ }^{7} \mathrm{Li}$ ratio (about $0.1)$ in this case. When $x \lesssim 1 \times 10^{-3}$, the ${ }^{11} \mathrm{~B} /{ }^{7} \mathrm{Li}$ ratio is almost equal to that of the mechanical mixing and when $x \gtrsim 1 \times 10^{-2}$ the ${ }^{11} \mathrm{~B} /{ }^{7} \mathrm{Li}$ ratio agrees with the ${ }^{11} \mathrm{C} /{ }^{7} \mathrm{Be}$ ratio of the mechanical mixing. In the latter case, both ${ }^{7} \mathrm{Li}$ and ${ }^{11} \mathrm{~B}$ are completely decomposed.

Now, we consider how the ${ }^{11} \mathrm{~B} /{ }^{7} \mathrm{Li}$ ratio depends on the location of the H-component in the mixture. Figure 10 presents the ${ }^{11} \mathrm{~B} /{ }^{7} \mathrm{Li}$ ratio in the mixtures for various $M_{m}(\mathrm{H})$. The location of the He-component is fixed to be $M_{m}(\mathrm{He})=$ 6.0 $M_{\odot}$. From this figure we see the following two characters. One is that the mixing ratio which gives the maximum ${ }^{11} \mathrm{~B} /{ }^{7} \mathrm{Li}$ ratio becomes larger as $M_{m}(\mathrm{H})$ increases. The reason is that the maximum temperature of the mixture decreases with an increase in $M_{m}(\mathrm{H})$, so that more protons need to decompose ${ }^{7} \mathrm{Li}$ and ${ }^{11} \mathrm{~B}$ in the mixture. The other is that the peak value of the ${ }^{11} \mathrm{~B} /{ }^{7} \mathrm{Li}$ ratio becomes larger as $M_{m}(\mathrm{H})$ increases as long as we are concerned with the case of $M_{m}(\mathrm{H}) \lesssim 7.2 M_{\odot}$, whereas it becomes smaller when $M_{m}(\mathrm{H}) \gtrsim 7.2 M_{\odot}$. This is brought about by the fact that ${ }^{7} \mathrm{Li}$ is more fragile than ${ }^{11} \mathrm{~B}$ to proton captures. When $M_{m}(\mathrm{H}) \lesssim 7.2 M_{\odot}$, a part of ${ }^{11} \mathrm{~B}$ and all ${ }^{7} \mathrm{Li}$ are decomposed in the mixture. The amount of the decomposed ${ }^{11} \mathrm{~B}$ becomes smaller with an increase in $M_{m}(\mathrm{H})$. When $M_{m}(\mathrm{H}) \gtrsim 7.2$ $M_{\odot}$, all ${ }^{11} \mathrm{~B}$ remains and a part of ${ }^{7} \mathrm{Li}$ is decomposed in the mixture. Increase in the final amount of ${ }^{7} \mathrm{Li}$ causes the decrease of ${ }^{11} \mathrm{~B} /{ }^{7} \mathrm{Li}$ ratio. At last, in the case of such a distant mixing where $M_{m}(\mathrm{H}) \gtrsim 8.4 M_{\odot}$ there does not occur the 


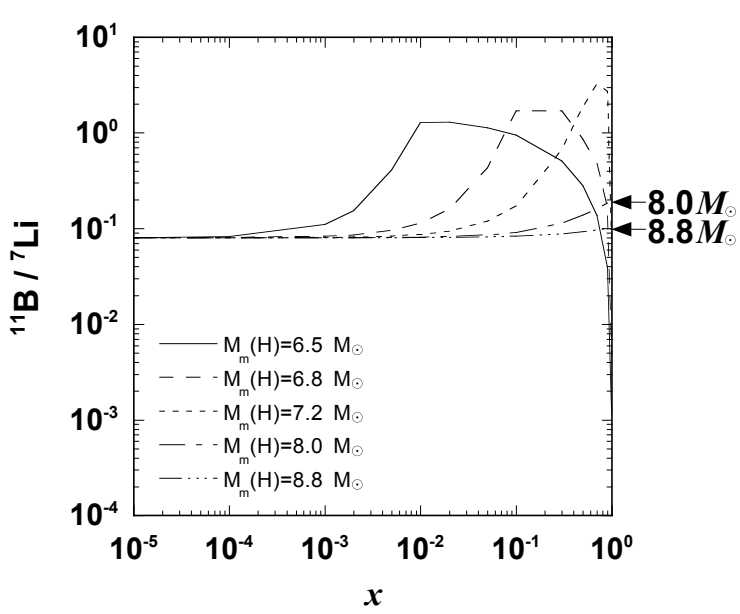

Fig. 10. The ${ }^{11} \mathrm{~B} /{ }^{7} \mathrm{Li}$ ratios for various $M_{m}(\mathrm{H})$ as a function of the mixing ratio, $x$. The location of the He-component is fixed to be $M_{m}(\mathrm{He})=6.0 M_{\odot}$. Arrows show the maximum ${ }^{11} \mathrm{~B} /{ }^{7} \mathrm{Li}$ ratios in the cases of $M_{m}(\mathrm{H})=8.0 M_{\odot}$ and $8.8 M_{\odot}$.

nuclear reprocessing on ${ }^{7} \mathrm{Li}$ and ${ }^{11} \mathrm{~B}$ after the mixing, so that the ${ }^{11} \mathrm{~B} /{ }^{7} \mathrm{Li}$ ratio is determined by the mechanical mixing.

Anyway, we find that the ${ }^{11} \mathrm{~B} /{ }^{7} \mathrm{Li}$ ratio is confined within a level between $1 \times 10^{-4}$ and 4 even if we take account of the nuclear reprocessing after the mixing; the ${ }^{11} \mathrm{~B} /{ }^{7} \mathrm{Li}$ ratio varies slightly wider than that evaluated in Paper I and $\mathrm{Pa}$ per II, i.e., $1 \times 10^{-4} \lesssim^{11} \mathrm{~B} /{ }^{7} \mathrm{Li} \lesssim 3$ but the difference is not so important.

\section{Summary and Conclusions}

We have investigated the varieties of the isotopic/elemental ratios of the light elements due to various parameters which would affect the nucleosynthesis of the light elements. In this study, we concentrated on the mixing process during the supernova explosion and explored the nuclear reprocessing on the light elements after the mixing. Four results are obtained.

1) The $X$-elements produced in the He-layer are decomposed by protons in the H-rich envelope when fluid components from these layers mix. The degree of the decomposition depends on the location of each component before the mixing, the mixing ratio, and the mixing time.

2) Among the $X$-elements, ${ }^{7} \mathrm{Li}$ and ${ }^{11} \mathrm{~B}$ which are abundant in the He-component are decomposed by only a small amount of protons from the H-component in the mixture. However, their $p$-rich isobars, ${ }^{7} \mathrm{Be}$ and ${ }^{11} \mathrm{C}$, are not decomposed. As a result, the variety of the ${ }^{11} \mathrm{~B} /{ }^{7} \mathrm{Li}$ ratio is not so changed even if the nuclear reprocessing after the mixing is considered.

3) The fragile species, ${ }^{6} \mathrm{Li}$ and ${ }^{9} \mathrm{Be}$, are completely decomposed by protons in the mixture if the mixing ratio is larger than $2 \times 10^{-3}$. Although ${ }^{10} \mathrm{~B}$ is also decomposed by protons in the mixture, the degree of the decomposition is less effective compared with ${ }^{6} \mathrm{Li}$ and ${ }^{9} \mathrm{Be}$.

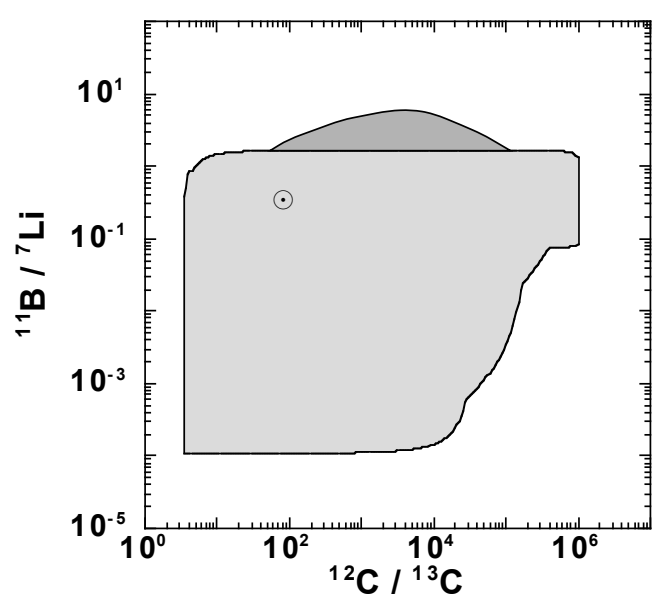

Fig. 11. The number ratios of ${ }^{11} \mathrm{~B} /{ }^{7} \mathrm{Li}-{ }^{12} \mathrm{C} /{ }^{13} \mathrm{C}$. The light shaded region denotes the ratios of the mechanical mixing between the He-layer and the H-rich envelope; the region is identical to the entire region shown in figure 14(a) in Paper I. The dark shaded region denotes the ratios not due to the mechanical mixing but due to the nuclear reprocessing after the mixing. The mark $\odot$ shows the ratios of the solar-system composition.

4) The abundances of the CNO-elements are scarcely affected by the nuclear reprocessing after the mixing.

In Paper I and Paper II, we investigated the possible ranges of the isotopic/elemental ratios, which we are interested in, using the three kinds of diagrams: ${ }^{11} \mathrm{~B} /{ }^{7} \mathrm{Li}^{-12} \mathrm{C} /{ }^{13} \mathrm{C}$, ${ }^{14} \mathrm{~N} /{ }^{15} \mathrm{~N}-{ }^{12} \mathrm{C} /{ }^{13} \mathrm{C}$, and ${ }^{16} \mathrm{O} /{ }^{17} \mathrm{O}-{ }^{12} \mathrm{C} /{ }^{13} \mathrm{C}$. In this study we find that the nuclear reprocessing after the mixing does not affect the abundances of the $\mathrm{CNO}$-elements. Thus, the diagrams of ${ }^{14} \mathrm{~N} /{ }^{15} \mathrm{~N}-{ }^{12} \mathrm{C} /{ }^{13} \mathrm{C}$ and ${ }^{16} \mathrm{O} /{ }^{17} \mathrm{O}-{ }^{12} \mathrm{C} /{ }^{13} \mathrm{C}$ are not changed even if the nuclear reprocessing is considered. Hence, we will mention only the diagram of ${ }^{11} \mathrm{~B} /{ }^{7} \mathrm{Li}-{ }^{12} \mathrm{C} /{ }^{13} \mathrm{C}$ ratios. In order to compare the ${ }^{11} \mathrm{~B} /{ }^{7} \mathrm{Li}^{12}{ }^{12} \mathrm{C} /{ }^{13} \mathrm{C}$ diagrams of the two studies, the present study and Paper I, we take account of the variety of the neutrino emission model as we did in Paper I: four neutrino emission models, given by Eqs. (3) and (4), are adopted additionally. The result is shown in Fig. 11. In addition to the light shaded region obtained by the mechanical mixing identical to the entire region shown in figure 14(a) in Paper I, there appears newly a dark shaded region in the ranges of $2 \lesssim^{11} \mathrm{~B} /{ }^{7} \mathrm{Li} \lesssim 6$ and $60 \lesssim^{12} \mathrm{C} /{ }^{13} \mathrm{C} \lesssim 1 \times 10^{5}$ owing to the effect of the nuclear reprocessing. Scatters of the elemental ratios due to the nuclear reprocessing are almost masked by those of the mechanical mixing and, as a result, the dark shaded region is apparently small compared with the region of the mechanical mixing. So, the diagram of ${ }^{11} \mathrm{~B} /{ }^{7} \mathrm{Li}-{ }^{12} \mathrm{C} /{ }^{13} \mathrm{C}$ ratios is scarcely changed even if we consider the nuclear reprocessing after the mixing.

From the above results we can say that the conclusions obtained in Paper I and Paper II are valid without large modifications even if we consider the nuclear reprocessing after the mixing between the He-layer and the H-rich envelope. The conclusions are as follows:

1) Presolar grains from the supernova should have the ${ }^{6} \mathrm{Li} /{ }^{7} \mathrm{Li}$ ratio less than $3 \times 10^{-5}$ and the ${ }^{9} \mathrm{Be} /{ }^{7} \mathrm{Li}$ ratios less than $2 \times 10^{-4}$. 
2) The ${ }^{11} \mathrm{~B} /{ }^{7} \mathrm{Li}$ ratio of the presolar grains from the supernova falls on the light shaded region or the dark shaded region in Fig. 11, which is almost the same as that of Paper I.

Acknowledgments. The authors would like to thank Kohichi Iwamoto, Ken'ichi Nomoto, and Toshikazu Shigeyama for giving the data of internal structure for a progenitor model, 14E1, and helpful discussion. This work is supported, in part, by Grand-inAid for General Scientific Research (B) (No. 09440089) and Research Fellowships of the Japan Society for the Promotion of Science for Young Scientists (No. 12000289). The computation has been made by Cray C916 and NEC SX-5 at the Computer Center of Tokyo Institute of Technology.

\section{References}

Amari, S., P. Hoppe, E. Zinner, and R. S. Lewis, Interstellar SiC with unusual isotopic compositions: grains from a supernova?, Astrophys. J., 394, L43-L46, 1992.

Amari, S., E. Zinner, and R. S. Lewis, Large ${ }^{18} \mathrm{O}$ excesses in circumstellar graphite grains from the Murchison meteorite: indication of a massivestar origin, Astrophys. J., 447, L147-L150, 1995.

Arnett, W. D. and A. Fu, The late behavior of supernova 1987A. I. The light curve, Astrophys. J., 340, 396-413, 1989.

Burrows, A. and B. A. Fryxell, An instability in neutron stars at birth, Science, 258, 430-434, 1992.

Burrows, A., J. Hayes, and B. A. Fryxell, On the nature of core-collapse supernova explosions, Astrophys. J., 450, 830-850, 1995.

Chandrasekhar, S., Hydrodynamic and Hydromagnetic Stability, pp. 428 436, Dover, New York, 1981.

Chevalier, R. A., The hydrodynamics of Type II supernovae, Astrophys. J., 207, 872-887, 1976.

Ebisuzaki, T., T. Shigeyama, and K. Nomoto, Rayleigh-Taylor instability and mixing in SN 1987A, Astrophys. J., 344, L65-L68, 1989.

Fryxell, B., E. Müller, and D. Arnett, Instabilities and clumping in SN 1987A. I. Early evolution in two dimensions, Astrophys. J., 367, 619634, 1991.

Fu, A. and W. D. Arnett, The late behavior of supernova 1987A. II. Gamma-ray transparency of the ejecta, Astrophys. J., 340, 414-425, 1989.

Hachisu, I., T. Matsuda, K. Nomoto, and T. Shigeyama, Nonlinear growth of Rayleigh-Taylor instabilities and mixing in SN 1987A, Astrophys. J., 358, L57-L61, 1990.

Hachisu, I., T. Matsuda, K. Nomoto, and T. Shigeyama, Mixing in ejecta of supernovae. I. General properties of two-dimensional Rayleigh-Taylor instabilities and mixing width in ejecta of supernovae, Astrophys. J., 390, 230-252, 1992.

Hachisu, I., T. Matsuda, K. Nomoto, and T. Shigeyama, Mixing in ejecta of supernovae. II. Mixing width of 2D Rayleigh-Taylor instabilities in the helium star models for type Ib/Ic supernovae, Astron. Astrophys. Suppl., 104, 341-364, 1994.

Herant, M. and S. E. Woosley, Postexplosion hydrodynamics of supernovae in red supergiants, Astrophys. J., 425, 814-828, 1994.

Herant, M., W. Benz, and S. Colgate, Postcollapse hydrodynamics of SN 1987A: two-dimensional simulations of the early evolution, Astrophys. $J ., 395,642-653,1992$.

Hoffman, R. D. and S. E. Woosley, Tables of reaction rates for nucleosynthesis for charged particle, weak, and neutrino interactions ( $Z<$ 45), (unpublished; http://isotopes.lbl.gov/isotopes / hw92_1.html), 1992.

Meyer, B. S., T. A. Weaver, and S. E. Woosley, Isotopic source table for a $25 M_{\odot}$ supernova, Meteoritics, 30, 325-334, 1995.

Nittler, L. R., S. Amari, E. Zinner, S. E. Woosley, and R. S. Lewis, Extinct ${ }^{44} \mathrm{Ti}$ in presolar graphite and $\mathrm{SiC}$ : proof of a supernova origin, Astrophys. J., 462, L31-L34, 1996.

Sedov, L. I., Similarity and Dimensional Methods in Mechanics, pp. 260270, Academic Press, New York and London, 1959.

Shigeyama, T. and K. Nomoto, Theoretical light curve of SN 1987A and mixing of hydrogen and nickel in the ejecta, Astrophys. J., 360, 242-256, 1990.

Thielemann, F.-K., M. Hashimoto, and K. Nomoto, Explosive nucleosynthesis in SN 1987A. II. Composition, radioactivities, and the neutron star mass, Astrophys. J., 349, 222-240, 1990.

Thielemann, F.-K., K. Nomoto, and M. Hashimoto, Core-collapse supernovae and their ejecta, Astrophys. J., 460, 408-436, 1996.

Travaglio, C., R. Gallino, S. Amari, E. Zinner, S. E. Woosley, and R. S. Lewis, Low-density graphite grains and mixing in Type II supernovae, Astrophys. J., 510, 325-354, 1999.

Wooden, D. H., Observational evidence for mixing and dust condensation in core-collapse supernovae, in Astrophysical Implications of the Laboratory Study of Presolar Materials, edited by T. J. Bernatowicz and E. Zinner, pp. 317-376, AIP Conference Proceedings 402, Woodbury, New York, 1997.

Woosley, S. E. and T. A. Weaver, The evolution and explosion of massive stars. II. Explosive hydrodynamics and nucleosynthesis, Astrophys. J. Suppl., 101, 181-235, 1995.

Woosley, S. E., D. H. Hartmann, R. D. Hoffman, and W. C. Haxton, The v-process, Astrophys. J., 356, 272-301, 1990.

Yoshida, T., Light elements synthesized in the He-layer and the H-rich envelope of Type II supernovae, Doctoral Thesis, 2000.

Yoshida, T., H. Emori, and K. Nakazawa, Light elements synthesized in the He-layer and the H-rich envelope of a type II supernova-Influence of the adopted neutrino emission model-, Earth Planets Space, 52, 203219, 2000a (Paper I).

Yoshida, T., K. Nakazawa, and H. Emori, Light elements synthesized in the He-layer and the H-rich envelope of a type II supernova, II-Influence of initial chemical compositions-, Earth Planets Space, 52, 361-376, 2000b (Paper II).

Zinner, E., Stellar nucleosynthesis and the isotopic composition of presolar grains from primitive meteorites, Annu. Rev. Earth Planet. Sci., 26, 147$188,1998$.

T. Yoshida (e-mail: tyoshida @gemini.rc.kyushu-u.ac.jp), H. Emori, and K. Nakazawa 